
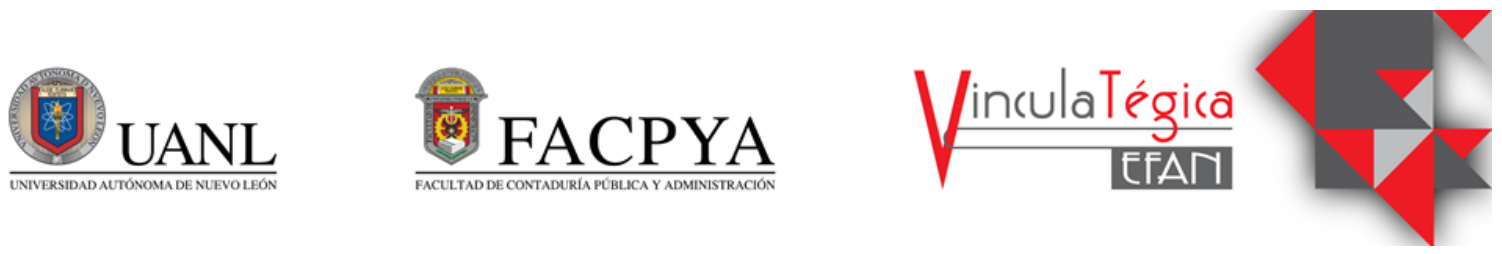

\title{
Análisis del riesgo de precio de las acciones mediante la estimación del Coeficiente beta $(\beta)$ de los bancos en México
}

\author{
César Camarillo Angeles ${ }^{1}$, Martha del Pilar Rodríguez García ${ }^{2}$ y Héctor Horacio Garza \\ Sánchez ${ }^{3}$ \\ IUniversidad Autónoma de Nuevo León, hugocesar.camarillo@gmail.com, Ciudad Universitaria, \\ 8113404430 \\ ${ }^{2}$ Universidad Autónoma de Nuevo León, martha.rodriguezgc@uanl.edu.mx, Ciudad Universitaria, \\ 8113404430 \\ ${ }^{3}$ Universidad Autónoma de Nuevo León, hector.garzasc@uanl.edu.mx, Ciudad Universitaria, \\ 8113404430
}

Información del artículo revisado por pares

Fecha de aceptación: junio-2021

Fecha de publicación en línea: diciembre-2021

DOI: https://doi.org/10.29105/vtga7.2-19

\section{Resumen}

Esta investigación se centró en la inversión en acciones y en el estudio del riesgo de mercado, al cual se refiere al riesgo de precio de las acciones y que este se analizó con más detalle porque contiene dos clasificaciones de gran importancia para una buena toma de decisiones a la hora de invertir en este tipo de instrumento y/o activo financiero llamado las acciones.

Además, en esta investigación se analizaron las acciones de las instituciones de banca múltiple en México y con ayuda del principal índice de la Bolsa Mexicana de Valores, el índice S\&P/BMV IPC, se estimó la medida de riesgo mejor conocida como el coeficiente beta, esto con el fin de conocer el impacto significativo que trae consigo el riesgo de mercado que es el riesgo de precio de las acciones, el cual si este riesgo es analizado y considerado de manera correcta puede ser de gran beneficio y ventaja al momento de invertir en este tipo de inversión.

Este estudio también se convierte en una guía para los interesados en la inversión en acciones para que puedan entender el mercado financiero al que pertenece este activo financiero llamado acciones, así como el sistema financiero mexicano de una manera general, lo cual es de gran importancia para conocer la zona a la que pertenece el sector bancario, que son las instituciones de banca múltiple, y el sector bursátil (Mercado de valores)

\section{Abstract}

This research focused on the investment in stocks and on the study of market risk, which refers to the equity price risk and which was analyzed in more detail because it contains two classifications of great importance for good decision making when investing in this type of instrument and/or financial asset called stocks.

In addition, in this research the stocks of multiple banking institutions in Mexico were analyzed and with the help of the main index of the Mexican Stock Exchange, the S\&P/BMV IPC index, the risk measure better known as the beta coefficient was estimated, this in order to know the significant impact that brings with it the market risk that is the equity price risk, which if this risk is analyzed and considered in the correct way can be of great benefit and advantage when investing in this type of investment.

This study also becomes a guide for those interested in stock investment so that they can understand the financial market to which this financial asset called stocks belongs, as well as the Mexican financial system in a general way, which is of great importance to know the zone to which the banking sector belongs, which are the multiple banking institutions and the stock market sector to 
al que pertenecen las empresas que cotizan en la Bolsa de Valores, como son las instituciones de banca múltiple, ya que estas ofrecen sus acciones, y que además, en la Bolsa Mexicana de Valores contiene el índice S\&P/BMV IPC, que fue un elemento esencial, junto con las acciones de las instituciones de banca múltiple, para la estimación del coeficiente beta en esta investigación.

Palabras clave: Acciones, Bancos, Coeficiente beta, Inversión, Mercado de valores y Riesgo.

Códigos JEL: B1, G1, G3.

\section{INTRODUCCIÓN}

Esta investigación tiene el objetivo de analizar la inversión en las acciones de las instituciones de banca múltiple en México, así como el riesgo de precio de acciones a las que estas se enfrentan en el mercado. Para este estudio no se utiliza una teoría tal cual, se utiliza más bien una medida de volatilidad de un activo financiero que es el coeficiente beta $(\beta)$, y que para esta investigación el activo financiero será las acciones de las instituciones de banca múltiple en México con respecto al índice S\&P/BMV IPC.

Esta medida del coeficiente beta $(\beta)$ ayuda a medir el grado de variabilidad del rendimiento de las acciones con relación al rendimiento del mercado que para esta investigación es el índice de la Bolsa Mexicana de Valores el S\&P/BMV IPC. Así como lo menciona el autor Vila (2016) el coeficiente beta $(\beta)$ mide qué tan sensible es la variación de un activo financiero frente a los cambios del mercado.

Es por eso que una vez que se haya calculado el coeficiente beta $(\beta)$ en esta investigación, se analizan dos variables fundamentales, por un lado, está la variable dependiente que es el coeficiente beta $(\beta)$ de las acciones de las instituciones de banca múltiple en México con respecto al índice S\&P/BMV IPC, el cual asimismo el índice S\&P/BMV IPC es un indicador referente del mercado de capitales de la BMV que es en donde se concentra todas las acciones de las empresas que cotizan en esta bolsa de valores y es un indicador representativo del mercado bursátil mexicano. Por otro lado, está la variable independiente que para fines de esta investigación será del tipo de riesgo de mercado el cual se refiere al riesgo de precio de las acciones, y que este mismo riesgo a su vez se divide en: riesgo diversificable y riesgo no diversificable.

Por lo cual se analiza el impacto de la variable independiente con la variable dependiente con la finalidad de identificar los aspectos y/o características which the companies listed in the Stock Exchange belong, such as the multiple banking institutions, as they offer their stocks, and moreover, in the Mexican Stock Exchange contains the S\&P/BMV IPC index, which was an essential element, together with the stocks of the multiple banking institutions, for the estimation of the beta coefficient in this research.

Keywords: Stocks, Banks, Beta coefficient, Investment, Stock market and Risk.

\section{JEL Codes: B1, G1, G3.}

que hacen que el coeficiente beta $(\beta)$ de las acciones de las instituciones de banca múltiple en México con respecto al índice S\&P/BMV IPC se mueva obteniendo variaciones negativas en los precios de las acciones y asimismo conocer y ayudar a mitigar este tipo de riesgo. Además, la investigación tiene por finalidad calcular el coeficiente beta $(\beta)$ de las acciones de las instituciones de banca múltiple en México con respecto al índice de la Bolsa Mexicana de Valores, el S\&P/BMV IPC, y posteriormente hacer un estudio general de las variaciones más notables que ha tenido la acción de cada institución de banca múltiple enfocado en el tipo de riesgo de mercado el cual es el riesgo de precio de las acciones que como se mencionó anteriormente este mismo riesgo se clasifica en: Riesgo diversificable y Riesgo no diversificable. También, se realizará una comparación de los coeficientes beta $(\beta)$ de cada una de las acciones de esta investigación.

La contribución que trae consigo la investigación será el crear conciencia al inversionista para que tome la mejor decisión de invertir en el sector bancario, así como la de evaluar la acción con relación al tipo de riesgo de mercado que es el riesgo de precio de las acciones.

Este trabajo proveerá una noción y asesoría para saber cómo invertir mejor en este instrumento financiero (acciones) ya que en ocasiones es la falta de análisis y desconocimiento lo que ocasiona que se termine invirtiendo mal el dinero.

$Y$ es que al momento de invertir en el mercado de valores se tiene que tomar en cuenta el nivel del riesgo y el rendimiento que debe tener el instrumento financiero al cual se decida invertir.

Así también como se menciona anteriormente es interesante el tema porque va dirigido a un sector que es muy atractivo para los inversionistas, así como su grado de importancia y el papel que cumple en la economía del país. Es por eso qué esta investigación va 
dirigido al sector bancario por ser uno de los más importantes en el desarrollo del país.

\section{MARCO TEÓRICO}

La investigación tiene como finalidad estudiar y analizar el impacto del riesgo de precio de las acciones en el coeficiente beta $(\beta)$ de las acciones de las instituciones de banca múltiple en México con respecto al índice S\&P/BMV IPC.

Asimismo, se identifica y se analiza el riesgo de precio de las acciones de las instituciones y lo que ocasiona que el rendimiento del precio de este activo financiero varié en el mercado.

La hipótesis general de la investigación es la siguiente: ¿el riesgo de precio de las acciones tiene una gran influencia en el valor del coeficiente beta $(\beta)$ de las acciones de las instituciones de banca múltiple en México con respecto al índice S\&P/BMV IPC? beta $(\beta)$ :

Las ventajas y desventajas del uso del coeficiente

- Permite medir la variabilidad de la rentabilidad de las acciones en el mercado de valores.

- Es útil para determinar el riesgo de mercado a corto plazo.

- Es una forma sencilla y rápida de realizar el cálculo.

- Es una medida que se integra en el CAPM.

- Se basa en datos históricos y no es precisamente un indicador de exactitud.

- Hay que tener en cuenta que es sólo una medida y que cada decisión depende del inversor.

- Es menos útil para determinar el riesgo de mercado a largo plazo, debido al factor de la volatilidad al paso de los años y por factores del mercado y de la empresa.

- No es la única forma de medir el riesgo de mercado.

\subsection{Marco teórico de la variable dependiente de la investigación}

El coeficiente beta $(\beta)$ de las acciones de las instituciones de banca múltiple en México con respecto al índice S\&P/BMV IPC. Dicho lo anterior de una manera escindida: ' La variabilidad del rendimiento de las acciones de las instituciones de banca múltiple en México con respecto al rendimiento del índice S\&P/BMV IPC',

Definiciones de la variable dependiente $\mathrm{Y}(\mathrm{El}$ coeficiente beta $(\beta)$ de las acciones de las instituciones de banca múltiple en México con respecto al índice S\&P/BMV IPC). La beta, una cifra que mide el riesgo no diversificable, o de mercado. La beta indica cómo responde el precio de un título a las fuerzas del mercado. Cuanto más sensible sea el precio de un título a los cambios del mercado, mayor será la beta de ese título. La beta se obtiene relacionando los rendimientos históricos de un título con el rendimiento de mercado. $\mathrm{El}$ rendimiento de mercado es el rendimiento promedio de todas las acciones (o de una muestra grande). (Gitman, L. y Joehnk, M., 2009).

El elemento $Y$ que conforma la variable dependiente (El coeficiente beta $(\beta)$ de las acciones de las instituciones de banca múltiple en México con respecto al índice $\mathrm{S} \& \mathrm{P} / \mathrm{BMV} \mathrm{IPC}$ ) de esta investigación se trata del precio de la acción de las instituciones de banca múltiple en México, las cuales cotizan en la bolsa mexicana de valores.

Cabe mencionar que se refieren a: "Instituciones de Banca Múltiple" como lo son: Bancos, Grupos Financieros y Sociedades Controladoras. Según Banco de México (2020) las empresas tienen dos formas de necesitar capital (fuentes de financiamiento) una de ellas es por medio del préstamo en forma de créditos o títulos de deuda y la otra es mediante la emisión de capital nuevo. En la primera opción la empresa se ve obligada a pagar intereses a quien le presto el dinero y en la segunda opción esta la emisión de capital que consiste en que las empresas efectúan pagos a los inversionistas si esta genera utilidades.

La emisión de capital para las empresas resulta menos arriesgada, pero para los inversionistas más arriesgada. Asimismo, Banco de México (2020) define a las acciones como títulos que representan el capital que se invierte en una empresa y pueden tener diferentes características dependiendo del poder de votación que el poseedor de las acciones tenga en la empresa que haya decidido invertir su dinero.

Las acciones pueden clasificar de dos maneras:

Acciones comunes:

Según la Bolsa Mexicana de Valores (2020) las acciones comunes o también llamadas ordinarias, son aquellas que, dependiendo de los estatutos sociales de la emisora, no tienen calificación o preferencia alguna. Estas acciones tienen derecho a voto general interviniendo en todos los actos de la vida de la empresa (Algunos como elegir al consejo de administración o decidir las políticas de la empresa). Sólo tendrán derecho a dividendos después de que se haya cubierto a las acciones preferentes.

Acciones preferentes:

Las acciones preferentes según la Bolsa Mexicana de Valores (2020) son aquellas que gozan de ciertos derechos sobre las demás acciones que conforman el 
capital social de una empresa. Los beneficios se refieren normalmente a la prioridad de pago en el caso de liquidación, así como al cobro de dividendos. Se emite con un dividendo determinado que debe pagarse antes de que se paguen dividendos a los poseedores de acciones comunes. Por lo general las acciones preferentes no tienen derecho a voto.

El elemento Y que forma parte de la variable dependiente en esta investigación se refiere a las acciones de las instituciones de banca múltiple que cotizan en la bolsa mexicana de valores.

A continuación, se presentan las 10 acciones de las instituciones de banca múltiple que se estudian a lo largo de la investigación:

Y1: GFNORTE (Grupo Financiero Banorte, S.A.B de C.V.)

Y2: GFINBUR (Grupo Financiero Inbursa, S.A.B. de C.V.)

Y3: GFMULTI (Grupo financiero Multiva S.A.B. de C.V.)

Y4: BBVA (Banco Bilbao Vizcaya Argentaria, S.A.)

Y5: SAN (Banco Santander, S.A.)

Y6: BBAJIO (Banco Del Bajío, S.A., Institución de Banca Múltiple)

Y7: BSMX (Banco Santander México, S.A., Institución de Banca Múltiple, Grupo Financiero Santander)

Y8: R (Regional, S.A.B. de C.V.)

Y9: MONEX (Monex, S.A.B. de C.V.)

Y10: GENTERA (Gentera, S.A.B. de C.V.)

\subsection{Marco teórico de la variable independiente de la investigación}

Elemento X, parte del coeficiente Beta $(\beta)$ :

X1: S\&P/BMV IPC (Índice de Precios y Cotizaciones que representa al mercado de capitales de la Bolsa Mexicana de Valores). El índice de precios y cotizaciones (S\&P/BMV IPC) del mercado de capitales de la BMV forma parte de la variable dependiente de esta investigación. Este índice se concentra todas las acciones de las empresas que cotizan en la bolsa mexicana de valores y es el índice más representativo del mercado de capitales en México.

Del tipo de riesgo de mercado: Riesgo de precio de las acciones. Asimismo, el riesgo de precio de las acciones se clasifica en: Riesgo diversificable y riesgo no diversificable.

Definiciones de la variable independiente $\mathrm{X}$ (El riesgo de precio de las acciones: Riesgo diversificable y riesgo no diversificable)

La Bolsa Mexicana de Valores (2020) define el riesgo de mercado como el riesgo que afecta al tenedor y/o poseedor de cualquier tipo de valor, ante las fluctuaciones de precio generadas y/o ocasionadas por los movimientos normales del mercado.

El riesgo de precio de las acciones o riesgo de cotizaciones de acciones, Grupo Sura (2020) lo define como el riesgo que ocurre cuando el valor razonable de un instrumento financiero fluctúa como resultado de las variaciones en los precios de los activos de renta variable. Como lo expresa el autor Rodríguez (2015) el riesgo de precio de las acciones es la posibilidad que la compañía tenga un resultado negativo, en función del precio de las acciones que posea.

Por otro lado, para el diario económico: Expansion.com (2020) el riesgo de cotizaciones de acciones es el riesgo de pérdida debido a movimientos en los precios de las acciones. Asimismo, el riesgo de precio de las acciones se clasifica en: riesgo diversificable y riesgo no diversificable.

Para el Grupo Financiero BASE (2018) el riesgo no diversificable, o riesgo sistemático, es cuando intervienen factores fuera del control de la empresa en la que se invierte, como las fluctuaciones económicas y monetarias o las variables políticas o sociales. Por ello, todas las acciones son vulnerables a sufrir pérdidas y, en consecuencia, afectar la rentabilidad de los fondos de inversión y de los inversionistas individuales.

Según el Institute of Business \& Finance (2016) el riesgo diversificable y/o riesgo no sistemático, también conocido como riesgo específico, riesgo idiosincrásico, representa los riesgos de una empresa específica, como la administración, las ventas, la participación en el mercado, la eliminación de productos, los conflictos laborales, entre otros. Este tipo de riesgo es particular de un activo, un riesgo que puede eliminarse mediante la diversificación.

El riesgo de precio de las acciones es la variable independiente de la investigación y es uno de los riesgos que integran el riesgo de mercado. A continuación, se muestra un panorama general de la estructura del riesgo sistemático:

Maneras de minimizar y cubrir el riesgo de precios de las acciones

1. La diversificación para minimizar el riesgo de precios de las acciones

A pesar de otros tipos de riesgo, el riesgo de precio de las acciones puede reducirse, esto, mediante la técnica de mitigación más común que es la diversificación.

2. Los futuros y las opciones para cubrir el riesgo de precios de las acciones

Además, el autor Kenton (2020) menciona que el riesgo de los precios puede cubrirse mediante la compra de derivados financieros llamados futuros y opciones. Un 
contrato de futuros obliga a una parte a completar una transacción a un precio y fecha predeterminados. El comprador de un contrato debe comprar y el vendedor debe vender el activo subyacente al precio establecido, independientemente de cualquier otro factor. Una opción ofrece al comprador la oportunidad de comprar o vender el activo subyacente, dependiendo del contrato, aunque no está obligado a hacerlo. Tanto los productores como los consumidores pueden utilizar estos instrumentos para cubrir el riesgo de los precios. A un productor le preocupa que el precio baje y a un consumidor le preocupa que el precio suba.

$\mathrm{El}$ activo subyacente es el activo que sirve como valor de referencia a los instrumentos derivados. Este activo puede ser financiero (acciones, tasas de interés, divisas, etc.) o no financiero (oro, cobre, petróleo, etc.). En este caso, el activo subyacente correspondería a las acciones.

3. La venta corta para cubrir el riesgo de precios de las acciones

El autor Chen (2020) comenta que la venta corta es una estrategia de inversión o de negociación que especula con la caída del precio de una acción o de otros valores. Es una estrategia avanzada que sólo deberían llevar a cabo los traders e inversores experimentados.

La venta corta implica la venta de acciones en las que el vendedor no es propietario de las mismas. El operador, anticipando una reducción en el precio de las acciones debido al riesgo de precio, planea pedir prestado, vender, comprar y devolver las acciones.

\section{METODOLOGÍA DE LA INVESTIGACIÓN}

Para efectos de esta investigación se realizó un estudio del precio de las acciones de las instituciones de banca múltiple en México, se debe aclarar que cuando se menciona instituciones de banca múltiple se está refiriendo a: Bancos, Grupos Financieros y Sociedades Controladoras: ya que estos ofrecen las actividades de captación y colocación de recursos (dinero).

Asimismo, esta investigación se adentró hacia aquellas instituciones de banca múltiple que cotizan en la bolsa mexicana de valores (BMV), aunque se debe considerar que actualmente existe otra bolsa de valores llamada ' 'bolsa institucional de valores (BIVA)'" pero que en esta investigación solo se centró en la BMV por su trayectoria y representación en el mercado bursátil, así como el historial de cotizaciones y/o operaciones que ha tenido en el mercado mexicano desde hace tiempo.

Igualmente, de la bolsa mexicana de valores se tomó como referencia el índice de precios y cotizaciones (S\&P/BMV IPC) el cual representa al mercado de capitales de la misma bolsa de valores en mención. Sin embargo, también la nueva bolsa de valores, la bolsa institucional de valores (BIVA) cuenta con su índice que simboliza al mercado de capitales el cual se llama "FTSE BIVA" pero que para esta investigación se utilizó solamente el índice de precios y cotizaciones (S\&P/BMV IPC) de la BMV por la trayectoria e historial de precios y cotizaciones que ha ofrecido en el mercado bursátil de México.

Para la comprobación de la hipótesis se utilizó la medida de riesgo conocida como el coeficiente Beta $(\beta)$ de cada una de las acciones que son objeto de estudio de esta investigación estimada mediante el uso de la regresión lineal simple en Excel.

\subsection{Tipo de investigación}

La investigación es cualitativa y cuantitativa dado a que describe cualidades y/o características del riesgo de precio de las acciones en esta investigación, así como de las instituciones de banca múltiple en México y también utilizo información histórica de forma numérica para llevar a cabo el respectivo análisis de regresión.

De tipo cualitativa:

- Exploratoria porque se buscó indagar información relacionada al tema de estudio de las variables como el caso del rendimiento de las acciones de las instituciones de banca múltiple en México y el riesgo de precio de las acciones, así también casi no se encontró investigaciones acerca del tipo de estudio que se pretendió realizar ya que es un estudio a un problema que no está definido.

- Descriptiva porque se pretendió describir el comportamiento del rendimiento de las acciones de las instituciones de banca múltiple en México con relación al rendimiento del índice del mercado de capitales de la Bolsa Mexicana de Valores, el índice S\&P/BMV IPC bajo un estudio de riesgo de precio de las acciones.

De tipo cuantitativa:

Analítica porque se realizó un análisis entre dos variables, por un lado, la variable independiente (El riesgo de precio de las acciones) y por otro lado la variable dependiente (El coeficiente beta $(\beta)$ de las acciones de las instituciones de banca múltiple en México con respecto al índice S\&P/BMV IPC).

Prospectiva porque los análisis y aportes que surgieron de este estudio conducen hacia adelante y proyectando hacia el futuro para nuevas investigaciones.

Transversal porque para la realización de los respectivos análisis de los objetos (elementos) de esta 
investigación, se recopilaron los datos en un periodo de tiempo que en este caso es desde que empezaron a cotizar las acciones de las instituciones de banca múltiple en la Bolsa Mexicana de Valores con relación al índice S\&P/BMV IPC hasta el primer semestre del año (2020). Los datos históricos del precio de acción de las instituciones de banca múltiple fueron extraídos del sitio web: Investing.com.

Correlacional porque para la obtención de la variable dependiente de esta investigación se calculó la relación entre dos elementos (subvariables), el primer elemento X (El rendimiento del índice S\&P/BMV IPC) y el segundo elemento $\mathrm{Y}$ (El rendimiento del precio de las acciones de las instituciones de banca múltiple en México). Por lo cual con un análisis de regresión de estos datos se obtuvo el coeficiente beta $(\beta)$ que para fines de la investigación fue la variable dependiente.

\subsection{Diseño de investigación}

Para esta investigación se utilizó un estudio de instancia generadora de la misma y por lo tanto se realizó un análisis para medir el impacto de las variables que fueron estudiadas. Es decir, para la investigación no se requirió la realización de una codificación de datos, ya que se utilizó información histórica y pública.

\subsection{Definición del instrumento de medición}

Los tipos de instrumentos de medición que se utilizaron en la investigación:

1. Observación: Revisión de comportamientos y situaciones observables del precio de la acción de las instituciones de banca múltiple en México y sus respectivas variaciones y riesgos en el mercado.

2. Análisis de contenido: Revisión de contenidos, tales como las estrategias implementadas, las ventajas competitivas que tienen y las campañas de publicidad que fomentan las instituciones de banca múltiple en México para mantener el precio de su acción en un nivel óptimo.

3. Datos recolectados de otros investigadores: Revisión de documentación física y electrónica, así como registros públicos (informe anual, etc.).

4. Análisis de datos: Se utilizo esta técnica que consiste en el estudio de datos con la finalidad de sacar conclusiones sobre la información mediante el uso de la Estadística. Para asimismo realizar un respectivo análisis de datos con el rendimiento del precio de las acciones de las instituciones de banca múltiple en México en relación con el rendimiento del Índice S\&P/BMV IPC.

\subsection{Definición de universo, población y muestra}

El universo está conformado por 144 acciones financieras de empresas de origen nacional y extranjero que cotizan en la Bolsa Mexicana de Valores (BMV). La población es de 27 acciones financieras de entidades financieras de origen nacional y extranjero que cotizan en la BMV. La población de estudio son las acciones financieras de las empresas de origen nacional y extranjero que cotizan en la Bolsa Mexicana de Valores y que corresponden al sector de servicios financieros y al subsector de entidades financieras.

La muestra es de 10 acciones financieras de instituciones de banca múltiple unas de origen nacional y otras de origen extranjero que cotizan en la BMV. Las partidas objeto de estudio las cuales pertenecen al ramo de: Bancos Comerciales, Grupos Financieros y Sociedades Controladoras, lo cual dicha selección lo conformaron las instituciones de banca múltiple de origen nacional y extranjero que cotizan en la Bolsa Mexicana de Valores y que es la muestra fundamental para la realización de la investigación. Además, en la investigación se decidió no tomar como objeto de estudio a PROMECAP ACQUISITION COMPANY, S.A.B. DE C.V; GRUPO PROFUTURO, S.A.B. DE C.V. y VALUE GRUPO FINANCIERO, S.A.B. DE C.V. ya que no ofrecen los productos y servicios de una Institución de Banca Múltiple. Asimismo, de igual forma no se consideraron a INVEX CONTROLADORA, S.A.B. DE C.V. y CORPORACION ACTINVER, S.A.B. DE C.V. ya que al ser también Sociedades Controladoras que tienen en su dominio e integran a una Institución de Banca Múltiple y que además cotizan en la Bolsa Mexicana de Valores, según la misma BMV no se consideran del ramo de: Bancos, Grupos Financieros y Sociedades Controladoras de Servicios Diversificados. Según la Bolsa Mexicana de Valores estas dos Sociedades Controladoras entran en el ramo de Mercados Financieros por el tipo de actividad económica que realizan.

Tipo de muestreo que se utilizó para la selección de la muestra

No probabilístico ya que los elementos se seleccionaron de acuerdo con características específicas. En este caso se seleccionaron únicamente las acciones de las empresas que conforman las instituciones de banca múltiple que cotizan en la Bolsa Mexicana de Valores.

Y por conveniencia porque así conviene al estudio, dado a que los elementos se seleccionaron a base del conocimiento y juicio del investigador. Se seleccionaron las acciones de las instituciones de banca múltiple que cotizan en la Bolsa Mexicana de Valores, 
refiriéndose a esto como lo son: Bancos Comerciales, Grupos Financieros y Sociedades Controladoras.

\subsection{Método de Análisis}

El método estadístico que se consideró y que es el más apropiados para el análisis de los datos recolectados (Las acciones de las Instituciones de Banca Múltiple en México y el índice S\&P/BMV IPC) es por medio del Análisis de regresión lineal simple, para calcular el coeficiente beta $(\beta)$ de las acciones.

\section{RESULTADOS}

Con el estudio se consiguió analizar y entender más a fondo el riesgo de precio de las acciones, el cual causo que haya variaciones en el precio de las acciones de las instituciones de banca múltiple en México.

A los inversionistas les será de gran utilidad los resultados de este análisis para una buena toma de decisión cuando se decida invertir en acciones de las instituciones de banca múltiple en México.

Los resultados obtenidos en esta investigación y la información que se agregó a la misma se llevaron a cabo de manera ética y transparente.

Para el cálculo del coeficiente beta $(\beta)$ de las acciones de las Instituciones de Banca Múltiple en México con respecto al índice S\&P/BMV IPC se tomó en cuenta lo siguiente:

Fecha inicial: Desde que empezaron a cotizar las acciones en la BMV

Fecha final: Hasta el primer semestre del año 2020

En el anexo 1 se presentan los resultados generales.

\subsection{Interpretación de resultados generales}

Y1: GFNORTE (Grupo Financiero Banorte, S.A.B de C.V.): El valor en Beta positivo de 1.344443121 significa que GFNORTE fue un poco más sensible que el mercado, es decir, lo que le sucede al mercado, GFNORTE lo recibe un poco más de una vez. A una proporción de un $34 \%$ más que el mercado.

Y2: GFINBUR (Grupo Financiero Inbursa, S.A.B. de C.V.): El valor en Beta positivo de 0.751387197 significa que GFINBUR fue casi mayor a la mitad de sensible que el mercado, es decir, lo que le pasa al mercado, GFINBUR lo recibe un poco más de la mitad. A una proporción de un $25 \%$ menos que el mercado. Esto menos que con lo que pasa con GFNORTE.
Y3: GFMULTI (Grupo Financiero Multiva S.A.B. de C.V.): El valor en Beta negativo de -0.064914823 significa que GFMULTI fue mucho menos de la mitad de sensible que el mercado, es decir, lo que le pasa al mercado, GFMULTI le afecta mucho menos de la mitad a tal grado de casi no ser afectado por el mercado. En otras palabras, se mueve casi nada, pero en dirección contraria al mercado. A una proporción de un $106 \%$ menos que el mercado. Esto nada que ver con lo que pasa con GFNORTE y GFINBUR que estos si se mueven en la misma dirección que el mercado.

Y4: BBVA (Banco Bilbao Vizcaya Argentaria, S.A.): El valor en Beta positivo de 0.987052865 significa que BBVA fue aproximadamente igual que sensible que el mercado, es decir, lo que le pasa al mercado, BBVA lo recibe cerca de una vez. A una proporción de un $1 \%$ menos que el mercado. Esto menos que con lo que sucede con BBAJIO.

Y5: SAN (Banco Santander, S.A.): El valor en Beta positivo de 0.929543686 significa que SAN fue aproximadamente igual que sensible que el mercado, es decir, lo que le pasa al mercado, SAN lo recibe cerca de una vez. A una proporción de un $7 \%$ menos que el mercado. Esto menos que con lo que pasa con BBAJIO y BBVA.

Y6: BBAJIO (Banco Del Bajío, S.A., Institución de Banca Múltiple): El valor en Beta positivo de 1.32162351 significa que BBAJIO fue más sensible que el mercado, es decir, lo que le sucede al mercado, BBAJIO lo recibe poco más de una vez. A una proporción de un $32 \%$ más que el mercado.

Y7: BSMX (Banco Santander México, S.A., Institución de Banca Múltiple, Grupo Financiero Santander): El valor en Beta positivo de 0.684076257 significa que BSMX fue casi la mitad de sensible que el mercado, es decir, lo que le sucede al mercado, BSMX lo recibe aproximadamente a medias. A una proporción de un $32 \%$ menos que el mercado. Esto menos que con lo que sucede con BBAJIO, BBVA y SAN.

Y8: R (Regional, S.A.B. de C.V.): El valor en Beta positivo de 1.475914476 significa que REGIONAL fue demasiado sensible que el mercado, es decir, lo que le sucede al mercado, REGIONAL lo recibe más de una vez. A una proporción de un $48 \%$ más que el mercado. Esto menos que con lo que pasa con GENTERA.

Y9: MONEX (Monex, S.A.B. de C.V.): El valor en Beta positivo de 0.074430183 significa que MONEX 
fue mucho menos de la mitad de sensible que el mercado, es decir, lo que le pasa al mercado, MONEX le afecta mucho menos de la mitad a tal grado de casi no ser afectado por el mercado. En otras palabras, se mueve casi nada, pero en la misma dirección al mercado. A una proporción de un $93 \%$ menos que el mercado. Esto mucho menos que con lo que sucede con GENTERA y REGIONAL.

Y10: GENTERA (Gentera, S.A.B. de C.V.): El valor en Beta positivo de 2.075048002 significa que GENTERA fue dos veces más sensible que el mercado, es decir, lo que le pasa al mercado, GENTERA lo recibe al doble. A una proporción de un 107\% más que el mercado.

\subsection{Interpretación de resultados por categoría de Grupos Financieros}

Y1: GFNORTE (Grupo Financiero Banorte, S.A.B de C.V.): El valor de Beta de 1.344443121 que tiene GFNORTE fue el mayor en comparación con el Beta de GFINBUR con 0.751387197 y GFMULTI con 0.064914823. Siendo así Banorte el Grupo Financiero más afectado por las variaciones del mercado.

Y2: GFINBUR (Grupo Financiero Inbursa, S.A.B. de C.V.): El valor de Beta de 0.751387197 que tiene GFINBUR representa que el Grupo Financiero fue afectado un poco más de la mitad por las variaciones del mercado, esto, después del Beta de GFNORTE con 1.344443121 y antes del Beta de GFMULTI con 0.064914823 .

Y3: GFMULTI (Grupo Financiero Multiva S.A.B. de C.V.): El valor de Beta de -0.064914823 que tiene GFMULTI lo define como el Grupo Financiero menos afectado por el riesgo de mercado. Por lo tanto, esta acción fue menos riesgosa que el mercado, pero siendo así esta deducción su rendimiento esperado será bajo.

\subsection{Interpretación de resultados por categoría de Bancos Comerciales}

Y4: BBVA (Banco Bilbao Vizcaya Argentaria, S.A.): El valor de Beta de 0.987052865 que tiene BBVA lo cataloga como el segundo Banco comercial que recibe el impacto del mercado en consideración, es decir, los cambios que suceden en el mercado a BBVA le impactan por igual.
Y5: SAN (Banco Santander, S.A.): El valor de Beta de 0.929543686 que tiene SAN lo señala como el tercer Banco comercial de esta categoría en recibir los impactos del mercado en una proporción aproximadamente a una vez.

Y6: BBAJIO (Banco Del Bajío, S.A., Institución de Banca Múltiple): El valor de Beta de 1.32162351 que tiene BBAJIO lo define en esta categoría como el Banco comercial más afectado por los cambios del Mercado.

Y7: BSMX (Banco Santander México, S.A., Institución de Banca Múltiple, Grupo Financiero Santander): El valor de Beta de 0.684076257 que tiene BSMX lo evidencia como el cuarto y último Banco comercial de esta categoría que recibe el impacto de los cambios del mercado aproximadamente casi a la mitad o a medias.

\subsection{Interpretación de resultados por categoría de Sociedades Controladoras}

Y8: R (Regional, S.A.B. de C.V.): El valor de Beta de 1.475914476 que tiene REGIONAL lo define como la segunda Sociedad Controladora en tener un Beta considerable ya que los cambios del mercado le afectan un poco más a Regional.

Y9: MONEX (Monex, S.A.B. de C.V.): El valor de Beta de 0.074430183 que tiene MONEX lo ubica como la Sociedad Controladora menos afectada dentro de esta categoría ya que los cambios en el mercado le impactan solamente la mitad.

Y10: GENTERA (Gentera, S.A.B. de C.V.): El valor de Beta de 2.075048002 que tiene GENTERA la coloca como la Sociedad Controladora más afecta dentro de la categoría y lo que sucede en el mercado a GENTERA le impacta al doble. 
Asimismo, las acciones de las instituciones de banca múltiple en México por categoría, que dependiendo del riesgo del precio de las acciones son más arriesgadas y menos arriesgadas que el mercado, son las siguientes:

\section{A: Por categoría de Grupos Financieros:}

Grupo Financiero con valor del coeficiente beta ( $\beta$ ) Mayor: Grupo Financiero Banorte, S.A.B de C.V: El valor de Beta es de: 1.344443121

A1: El Valor de Beta en Banorte se debe a que por ser un Grupo Financiero "Grande" se encuentra sufriendo las consecuencias de tener un Beta así, el cual significa que el riesgo de mercado le está impactando a proporción de 1.

Grupo Financiero con valor del coeficiente beta ( $\beta$ ) Menor: Grupo Financiero Multiva S.A.B. de C.V: El valor de Beta es de: -0.064914823

A2: El Valor de Beta de Multiva es el único de las instituciones de banca múltiple que salió negativo, es decir la acción se mueve casi nada, pero en dirección contraria al mercado, siendo así está la acción menos riesgosa que el mercado en esta categoría, pero hay que tener en cuenta que cuando no hay riesgo, no hay rendimiento. Asimismo, Multiva pertenece al conglomerado del Grupo Empresarial Ángeles el cual es un grupo financieramente fuerte. Pero en sí, el valor de Beta de Multiva significa tener un menor nivel de rendimiento esperado.

\section{B: Por categoría de Bancos Comerciales:}

Banco Comercial con valor del coeficiente beta ( $\beta$ ) Mayor: Banco Del Bajío, S.A., Institución de Banca Múltiple: El valor de Beta es de: 1.32162351

B1: El Valor de Beta de BanBajío es normal que una institución de su tamaño tenga esos valores en Beta ya que su especialidad es la captación y colocación de créditos. En sí, su valor significa que cuando ocurran cambios en el mercado a BanBajío le afectara aproximadamente a una razón de 1.3, algo considerable. Asimismo, es una de las acciones más riesgosas que el mercado en esta categoría. Por lo tanto, sus rendimientos esperados tienden a ser altos, porque a mayor riesgo mayor rendimiento.
Banco Comercial con valor del coeficiente beta (ß) Menor: Banco Santander México, S.A., Institución de Banca Múltiple, Grupo Financiero Santander: El valor de Beta es de: 0.684076257

B2: El Valor de Beta de Santander México es interesante, debido a que por ser sociedades extranjeras el Beta les impacta la mitad las variaciones del mercado, es decir, cerca de un $50 \%$. Hay que tener en cuenta que Santander México le pertenece casi un 92\% del capital social a Santander Español. Asimismo, el valor de beta significa que lo que le sucede al mercado, Santander México lo recibe aproximadamente más de la mitad. También se pudiera decir que la acción es menos riesgosa que el mercado.

\section{C: Por categoría de Sociedades Controladoras:}

Sociedad Controladora con valor del coeficiente beta ( $\beta$ ) Mayor: Gentera, S.A.B. de C.V.: El valor de Beta es de: 2.075048002

C1: El Valor de Beta de Gentera (Compartamos Banco) es la que resulto con mayor impacto por las variaciones del mercado y es que compartamos banco es una microfinanciera que se dedica a otorgar créditos a pequeñas y medianas empresas y cuando ocurren cambios en el mercado por su capacidad siempre saldrá afectada, y se puede comprobar con lo que ha sucedido con esta actual pandemia del COVID-19 su acción es la más golpeada de todos por el hecho de que es una microfinanciera, asimismo para solucionar esto, compartamos bancos a pedido a sus propietarios que le aumenten su capital para poder solventar sus deudas y además seguir otorgando créditos a sus clientes. Incluso esta acción es la más riesgosa de todas las acciones de las instituciones de banca múltiple en México. Pero según la relación que hay entre rendimiento y riesgo, entre mayor sea el riesgo mayor será el rendimiento, y viceversa.

Sociedad Controladora con valor del coeficiente beta $(\beta)$ Menor: Monex, S.A.B. de C.V: El valor de Beta es de: 0.074430183

C2: El Valor de Beta de Monex es también interesante porque significa que las variaciones del mercado le afectan mucho menos de la mitad a tal grado de casi no ser afectado por el mercado, es decir, casi no se mueven sus rendimientos y por lo tanto el riesgo es menor. Asimismo, hay que tener en cuenta que los tipos 
de servicios financieros a que se dedica resaltan los cambios de divisas y las transacciones de pagos internacionales. Además, Hay que resaltar que Monex tiene presencia en países de gran economía.

Asimismo, dentro de lo que define a: "Instituciones de banca múltiple" se encuentran los: Bancos, Grupos Financieros y Sociedades Controladoras, los cuales responden de diferente manera a las variaciones del mercado debido a como estos están estructurados, enseguida se muestra un detalle general de estos:

- Por un lado, los Grupos Financieros es la agrupación de instituciones financieras que ofrecen diversos servicios. Para ser un Grupo Financiero se debe tener por lo menos tres instituciones financieras las cuales pueden ser: Banco, Casa de Bolsa, Aseguradora, Casa de Cambio, Almacenadora y Arrendadora, etcétera.

- Por otro lado, los Bancos Comerciales son entidades financieras cuya especialidad principal es el crédito, es decir, aceptan depósitos del público y estos mismos crean depósitos a la vista (prestamos). Además de que pueden ofrecer otros tipos de servicios financieros. Legalmente se le conoce como: Institución de banca múltiple. También una institución de banca múltiple puede ser llamada: Banco, Banca, Entidad de crédito, Entidad de depósito, etcétera.

- Asimismo, las Sociedades Controladoras como su nombre lo indica son sociedades que tienen el control de otras compañías y que poseen más del $50 \%$ del capital de las acciones de estas mismas. También una Sociedad Controladora puede ser llamada: Tenedora, Controladora, Holding, Matriz, etcétera.

Además, en la investigación se encontró que los Grupos Financieros son fuertes debido a que como se mencionó anteriormente es por su estructura organizacional y porque ofrecen diversos productos y servicios, además lo que se percato es que estos mismos tienen una estrecha relación con empresas y familias de alto reconocimiento:

1. Grupo Financiero Banorte: Pertenece a las familias González y Hank los cuales son dueños de la compañía global de alimentos GRUMA y de los negocios del Grupo Hermes, entre otros.

2. Grupo Financiero Inbursa: Pertenece a la familia Slim y los cuales son propietarios del Grupo Carso, América Móvil y Fundación Carlos Slim, entre otros.

3. Grupo Financiero Multiva: Pertenece a la familia Vázquez Raña y los cuales son propietarios del Grupo Empresarial Ángeles, entre otros.

Así también, los Bancos Comerciales son instituciones lo cual su fortaleza es en la especialización de la colocación y captación de créditos, también mencionar que estos fueron los que más recibieron los impactos de las variaciones del mercado por el hecho de dedicarse al servicio de préstamos al público.

Igualmente, las Sociedades Controladoras son instituciones interesantes debido a su estructura organizativa porque estas controlan a otras empresas, es decir, tienen en su dominio a otras sociedades, además las sociedades controladoras realizan determinadas actividades, así como poseer acciones de otras corporaciones.

El resultado de la hipótesis general de la investigación fue aceptado y se argumentó lo siguiente:

Por un lado, el riesgo de precio de las acciones, este tipo de riesgo de mercado que envuelve dos clasificaciones, una es el riesgo diversificable que este se relaciona a la toma de decisión del inversionista, el cual el riesgo diversificable se puede minimizar mediante la diversificación de la inversión en esta clase de activos financieros, es decir, invertir en otros sectores y no solamente en uno en específico, o también en dado caso optar por invertir en otros instrumentos financieros pero la finalidad en esta investigación es priorizar la inversión en el activo financiero de las: "acciones" por el rendimiento que pueden llegar a tener. $\mathrm{Y}$ otro es el riesgo no diversificable que este se relaciona con lo que esta fuera de control del inversionista y de la misma empresa a la cual se invierte, es decir, el riesgo no diversificable se refiere a las variaciones en los factores del mercado, en otras palabras, los factores que ocurren en el ámbito político, social y económico, entre otros. Asimismo, se puede deducir que el riesgo de precio de las acciones está conformado por un 50/50 una por parte del inversionista (decisiones) y la otra por parte del mismo mercado (condiciones).

Por otro lado, el coeficiente beta $(\beta)$, esta medida de riesgo en cuanto a las diferencias que se pueden encontrar en las Instituciones de banca múltiple 
también catalogadas y ubicadas en Bancos Comerciales, Grupos Financieros y Sociedades Controladoras se engloban factores que van desde su estructura organizacional, los productos y servicios que ofrecen, el tipo de actividad económica que realizan, entre otros. Por lo cual se puede deducir que el coeficiente beta $(\beta)$ varia debido a la categoría en la que se encuentre la institución de banca múltiple y su tamaño, además de sus características antes mencionadas.

\section{CONCLUSIONES}

La investigación lleva a cabo un análisis del coeficiente beta $(\beta)$ de las acciones de las instituciones de banca múltiple en México con respecto al índice $S \& P / B M V$ IPC bajo el estudio del tipo de riesgo de mercado: el riesgo de precio de las acciones, en donde se encontraron varias conclusiones, que se presentan a continuación:

Las diferencias que influyeron en el riesgo del precio de las acciones sobre el valor del coeficiente beta ( $\beta$ ) para cada una de las acciones de las instituciones de banca múltiple en México debido a que en esta investigación se entendió por: "Instituciones de banca múltiple" a: Bancos Comerciales, Grupos Financieros y Sociedades Controladoras fueron:

Las variaciones del precio de las acciones de las instituciones de banca múltiple en México se evidencian notablemente cuando ocurre los eventos inesperados de las crisis, en este tipo de situaciones los bancos salen más perjudicados ya que quedan expuestos a perdidas y no solo eso, sino que a nuevos cambios.

Las instituciones de banca múltiple en México luego de esta crisis sanitaria (COVID-19) optaran por formar alianzas con empresas de tecnología o desarrollar más aplicaciones como medio para ofrecer sus productos de una manera más eficiente y con satisfacción total a sus clientes.

Esta investigación más allá de ser una herramienta útil que puede ayudar a los inversionistas a realizar una buena toma de decisiones de inversión. También se vuelve como una guía que ayudara a los lectores de este estudio (sociedad interesada en temas de inversión) que todavía no invierten en la bolsa de valores, y así promover el interés de empezar a invertir, y a su vez a los inversionistas a tomar el riesgo de inversión de una manera más consciente y segura en el instrumento financiero (acciones).

Igualmente, contribuirá al crecimiento del mercado bursátil mexicano porque habrá mayor flujo de inversionistas y cuando hay un mayor interés por parte de este individuo fundamental, la economía del país empezará a fortalecerse bursátilmente porque las empresas que no cotizan en bolsa verán que el mercado nacional es atractivo y por ende empezaran a querer cotizar en alguna bolsa de valores. Recordemos el término de la ley de la oferta y la demanda, pero enfocada en el instrumento financiero (acciones), en sí, los individuos que hacen al mercado son los inversionistas y para que exista un mercado se necesita que haya un demandante interesado (inversionista) que quiera adquirir el producto (acciones), y por ende habrá empresas que se volverían emisoras de valores y que ofertaran sus instrumentos financieros en algún mercado bursátil y que estos serán adquiridos por la comunidad de inversionistas.

Con lo que se logra al realizar este trabajo de investigación es saber identificar los riesgos que conlleva realizar una inversión en acciones, examinar el panorama del mercado financiero e invertir sabiamente en el instrumento financiero de las acciones. También ayuda a concientizar para que se tenga la seguridad y confianza de que al invertir en este instrumento financiero no se está invirtiendo en algo desconocido, ya que el mercado financiero tiene sus propias leyes y autoridades que hacen que el mercado de valores este organizado y respaldado para así evitar algún tipo de fraude y/o actividad ilícita, es decir, resaltar que en la actualidad los mercados bursátiles son mercados seguros y confiables para invertir.

Mencionando por último, una limitación que se tuvo al llevar a cabo la investigación fue la obtención de los datos del precio de las acciones, debido a que los datos del precio de las acciones fueron extraídos del sitio: Investing.com, ya que hubo meses en que no se visualizaban las cotizaciones correspondientes de los precios de las acciones, pero que a pesar de esto, este obstáculo no influyó en los resultados y en la finalidad que se tenía contemplado llegar en este estudio, porque el coeficiente beta marco una tendencia realista del comportamiento actual que posee el precio de cada una de las acciones. 


\section{REFERENCIAS}

Vila, R. (2016). PKF Vila Naranjo y PKF Accountants and Business Advisors. Determinación del Coeficiente Beta $(\beta)$ o Riesgo no diversificable. Recuperado de: http://pkfperu.com/wpcontent/uploads/2016/05/Interpretando_25.pdf

Banco de México (2020). ¿Qué son las Acciones? http://www.anterior.banxico.org.mx/dyn/ley-detransparencia/consultas-frecuentes/existencia-comunicaciones-fra.html

Bolsa Mexicana de Valores (2020). Glosario. Recuperado de: https://www.bmv.com.mx/es/grupobmv/glosario

Gitman, L. y Joehnk, M. (2009). Fundamentos de inversiones. México: Pearson Educación.

Grupo Sura (2020). 2020 1T Estados financieros consolidados. Recuperado de: https://www.gruposura.com/wp-content/uploads/2020/05/grupo-sura-estados-financierosconsolidados-2020-1t.pdf

Rodríguez, I. (2015). Riesgo de Mercado. Lo que todo Auditor de saber. Recuperado de: https://www.auditool.org/blog/control-interno/3171-riesgo-de-mercado-lo-que-todo-auditorde-saber

Expansion.com. (2010). Glosario financiero. Recuperado de: https://www.expansion.com/actualidad/glosario/financiero/r.html\#rdpda

Institute of Business \& Finance. (2016). Systematic and Unsystematic Risk. Recuperado de: https://icfs.com/financial-knowledge-center/systematic-and-unsystematic-risk

Grupo Financiero BASE (2018). Inversión en acciones: Riesgos diversificables y no diversificables. Recuperado de: https://blog.bancobase.com/inversi\%C3\%B3n-en-acciones-riesgosdiversificables-y-no-diversificables

Kenton, W. (2020). Price Risk. Investopedia. Recuperado de: https://www.investopedia.com/terms/p/pricerisk.asp\#: :text=Price\%20risk\%20is\%20the\%20 risk $\% 20$ that $\% 20$ the $\% 20$ value $\% 20$ of $\% 20$ a,tool\%20to\%20mitigate\%20price\%20risk.

Chen, J. (2020). Short Selling. Investopedia. Recuperado de: https://www.investopedia.com/terms/s/shortselling.asp

Investing.com (2020). Grupo Financiero Banorte (GFNORTEO) - Información histórica. Recuperado de: https://mx.investing.com/equities/financiero-banorte-historical-data

Investing.com (2020). Grupo Financiero Inbursa, SAB De CV (GFINBURO) - Información histórica. Recuperado de: https://mx.investing.com/equities/grupo-financiero-inbursa-historical-data

Investing.com (2020). Grupo Financiero Multiva SAB De CV (GFMULTIO) - Información histórica. Recuperado de: https://mx.investing.com/equities/grupo-financiero-multiva-historical-data

Investing.com (2020). BBVA (BBVA) - Información histórica. Recuperado de: https://mx.investing.com/equities/bbva-historical-data?cid=26993

Investing.com (2020). Banco Santander (SAN) - Información histórica. Recuperado de: https://mx.investing.com/equities/banco-santander-historical-data?cid=26996

Investing.com (2020). Banco Del Bajio SA Institution De Banca Multiple (BBAJIOO) - Información histórica. Recuperado de: https://mx.investing.com/equities/banco-del-bajio-sa-historical-data

Investing.com (2020). Grupo Financiero Santander México SAB de CV Class B (BSMXB) Información histórica. Recuperado de: https://mx.investing.com/equities/grupo-financierosantander-b-historical-data

Investing.com (2020). BanRegio Grupo Financiero SAB de CV (RA) - Información histórica. Recuperado de: https://mx.investing.com/equities/banregio-grupo-financiero-o-historical-data

Investing.com (2020). Holding Monex SAB de CV (MONEXB) - Información histórica. Recuperado de: https://mx.investing.com/equities/holding-monex-sab-de-cv-historical-data

Investing.com (2020). Gentera SAB de CV (GENTERA) - Información histórica. Recuperado de: https://mx.investing.com/equities/compartamos-historical-data 


\section{ANEXOS}

Anexo 1: Resultados generales del coeficiente beta $(\beta)$ de las acciones de las Instituciones de Banca Múltiple en México con respecto al índice S\&P/BMV IPC.

\begin{tabular}{|c|c|c|c|c|}
\hline Elemento & $\begin{array}{c}\text { Clave } \\
\text { emisora }\end{array}$ & Razón social & Categoría & Valor de BETA \\
\hline$Y 1$ & GFNORTE & $\begin{array}{c}\text { GRUPO FINANCIERO BANORTE, S.A.B } \\
\text { DE C.V. }\end{array}$ & $\begin{array}{c}\text { Grupos } \\
\text { Financieros }\end{array}$ & 1.344443121 \\
\hline Y2 & GFINBUR & $\begin{array}{c}\text { GRUPO FINANCIERO INBURSA, S.A.B. } \\
\text { DE C.V. }\end{array}$ & $\begin{array}{l}\text { Grupos } \\
\text { Financieros }\end{array}$ & 0.751387197 \\
\hline Y3 & GFMULTI & $\begin{array}{c}\text { GRUPO FINANCIERO MULTIVA S.A.B. DE } \\
\text { C.V. }\end{array}$ & $\begin{array}{l}\text { Grupos } \\
\text { Financieros }\end{array}$ & -0.064914823 \\
\hline Y4 & $B B V A$ & $\begin{array}{c}\text { BANCO BILBAO VIZCAYA ARGENTARIA, } \\
\text { S.A. }\end{array}$ & $\begin{array}{l}\text { Bancos } \\
\text { Comerciales }\end{array}$ & 0.987052865 \\
\hline Y5 & $S A N$ & BANCO SANTANDER, S.A. & $\begin{array}{l}\text { Bancos } \\
\text { Comerciales }\end{array}$ & 0.929543686 \\
\hline Y6 & BВAJIO & $\begin{array}{c}\text { BANCO DEL BAJÍO, S.A., INSTITUCIÓN } \\
\text { DE BANCA MÚLTIPLE }\end{array}$ & $\begin{array}{l}\text { Bancos } \\
\text { Comerciales }\end{array}$ & 1.32162351 \\
\hline Y7 & $B S M X$ & $\begin{array}{l}\text { BANCO SANTANDER MEXICO, S.A., } \\
\text { INSTITUCION DE BANCA MULTIPLE, } \\
\text { GRUPO FINANCIERO SANTANDER }\end{array}$ & $\begin{array}{l}\text { Bancos } \\
\text { Comerciales }\end{array}$ & 0.684076257 \\
\hline Y8 & $R$ & REGIONAL, S.A.B. DE C.V. (BANREGIO) & $\begin{array}{l}\text { Sociedades } \\
\text { Controladoras }\end{array}$ & 1.475914476 \\
\hline Y9 & MONEX & $\begin{array}{l}\text { MONEX, S.A.B. DE C.V. }(\text { GRUPO } \\
\text { FINANCIERO MONEX) }\end{array}$ & $\begin{array}{l}\text { Sociedades } \\
\text { Controladoras }\end{array}$ & 0.074430183 \\
\hline Y10 & GENTERA & $\begin{array}{l}\text { GENTERA, S.A.B. DE C.V. } \\
\text { (COMPARTAMOS BANCO) }\end{array}$ & $\begin{array}{l}\text { Sociedades } \\
\text { Controladoras }\end{array}$ & 2.075048002 \\
\hline
\end{tabular}

Fuente: Elaboración propia. 